\title{
THE DISCHARGE CHARACTERISTICS OF VAGAL CARDIAC NEURONS DURING CLASSICALLY CONDITIONED HEART RATE CHANGE ${ }^{1}$
}

\author{
MICHAFI, R. GOLD ${ }^{*, 2}$ AND DAVID H. COHEN $\ddagger^{3}$ \\ ${ }^{*}$ Department of Physiology, University of Virginia School of Medicine, Charlottesuille, Virginia 22904 and $\ddagger$ Department of \\ Neurobiology and Behavior, State University of New York at Stony Brook, Stony Brook, New York 11794
}

Received December 19, 1983; Revised June 13, 1984; Accepted June 14, 1984

\begin{abstract}
Classically conditioned heart rate change in the pigeon has been developed as a vertebrate model system for cellular analysis of associative learning. One aspect of this development involved characterizing in detail the "final common path" for the conditioned response, the "cardiac motoneurons." With this as a foundation it has been possible to investigate the discharge properties of these motoneurons during acquisition of the conditioned heart rate response, and in this paper we report such data for the vagal (parasympathetic) component of the conditioned response.

The activity of single neurons was recorded in the intermediate zone of the dorsal motor nucleus of the vagus of the pigeon. Antidromic activation confirmed that these cells gave rise to efferent axons in the vagus nerve and allowed their classification on the basis of conduction velocity into three groups: A, B1 (cardiac), and B2 units. B1 and B2 units had low levels of irregularly maintained activity, whereas A units discharged more regularly at higher frequency. B1 units had a weak cardiac rhythm, no respiratory rhythm, and showed decreased discharge in response to visual stimulation or foot-shock. These stimulus-evoked decreases in the activity of cardiac cells were accompanied by increases in heart rate. B2 units were unresponsive to both visual stimulation and foot-shock, whereas A units were unresponsive to visual stimulation but possibly responsive to foot-shock.

The activity of vagal cardiac neurons was recorded during or after classical conditioning (paired lights and foot-shocks) or sensitization (unpaired lights and foot-shocks) to characterize discharge changes associated with conditioned cardioacceleration. The initial light presentation prior to training elicited a small cardioacceleratory response accompanied by a small decrease in neuronal discharge. This response was enhanced monotonically over conditioning but was attenuated over sensitization. Paralleling this, the cardioacceleratory response increased monotonically with conditioning and attenuated with unpaired lights and shocks. Furthermore, the response latencies were short and diverged as a function of associative or nonassociative training, decreasing over conditioning and increasing over sensitization.

These results confirm vagal involvement in conditioned heart rate change and demonstrate a number of important characteristics of this vagal contribution.
\end{abstract}

In recent years invertebrate model systems for the cellular and molecular analysis of learning have yielded exciting results (e.g., Alkon, 1982; Kandel and Schwartz, 1982). The development of vertebrate models is more challenging and has thus been understandably slower. Nevertheless, there is now a growing number of such models with considerable promise (Cohen, 1982; Thompson et al., 1982; Tsukahara, 1982).

\footnotetext{
${ }^{1}$ This investigation was supported by National Science Foundation Grants BNS-75-20537 and BNS-80-16396 to D. H. C. M. R. G. was supported in part by National Institutes of Health Training Grant T32 HLO7284. This paper is based in part upon a doctoral dissertation submitted to the University of Virginia by M. R. G. We thank Mr. Dennis Goff and Ms. Doris Hannum for their technical assistance.

${ }^{2}$ Present address: Department of Physiology, University of Colorado School of Medicine, Denver, CO 80262.

${ }^{3}$ To whom correspondence should be addressed.
}

A model involving visually conditioned heart rate change in the pigeon is particularly well developed (Cohen, 1974a, 1980, $1982,1984)$. This system has been characterized behaviorally in considerable detail, and a standardized paradigm for longterm associative learning has been established that is quite effective for cellular neurophysiological analysis (Cohen and Goff, 1978). Furthermore, substantial effort has been devoted to identifying the relevant neuronal circuitry, and this has generated a first approximation to a pathway from the eye to the heart that must be intact for acquisition of the conditioned response (CR) (Cohen, 1980).

An important aspect of this mapping effort was describing the "cardiac motoneurons" or "final common path" for the CR (Cohen, 1974b). A behavioral study involving different combinations of cardiac denervation and pharmacological blockade (Cohen and Pitts, 1968) established that the CR is mediated entirely by the extrinsic cardiac nerves, with both the vagal and sympathetic innervations participating. With respect to 
the parasympathetic innvervation, the cells of origin of the vagal cardiac fibers have been localized to a limited region of the dorsal motor nucleus of the vagus (DMN) (Cohen and Schnall, 1970; Cohen et al., 1970). Also, we have quantitatively described the fiber spectrum of the vagus and various of its branches, determined the compound action potential components associated with bradycardia, and established criteria for electrophysiologically identifying vagal cardiac neurons (Schwaber and Cohen, 1978a, b).

Such studies have established a foundation for characterizing the behavior of the vagal cardiac neurons during acquisition of the conditioned heart rate response. This is important for a number of reasons. First, it would allow a description of the $\mathrm{CR}$ in a neurophysiological time domain by eliminating delays associated with conduction to and transmission at the periphery (Warner and Cox, 1962; Cabot, 1976). This would permit estimation of the central processing time for the $\mathrm{CR}$ which, in turn, provides an assessment of how amenable the system is to neurophysiological analysis. Second, it would characterize more accurately the relative contributions of the vagal and sympathetic cardiac innervations to the CR. Finally, it would establish the basis for investigating more central components of the descending pathways involved in the expression of the CR.

In this paper we report experiments describing the activity of the vagal cardiac neurons during and after acquisition of a conditioned heart rate response (Gold, 1979; Gold and Cohen, 1981a). The activity of various vagal noncardiac neurons is also described.

\section{Materials and Methods}

\section{General procedures}

The experiments utilized 75 (male and female) White Carneaux pigeons (Columba livia) weighing 400 to $600 \mathrm{gm}$ and ranging in age from 2 to 6 months. All animals were housed individually, permitted free access to food and water, and remained on a 12-hr light-dark cycle for at least 2 weeks before experimentation.

Five to 15 days before recording, the posterior cerebellum was removed by aspiration under pentobarbital anesthesia $(25 \mathrm{mg} / \mathrm{kg})$ to allow visualization of the floor of the fourth ventricle; the cavity was then packed with Gelfoam and the wound was closed.

For neurophysiological experiments the skin overlying all incision points, as well as all pressure points, was liberally infiltrated with lidocaine. The trachea was intubated and immobilization was induced with $\alpha$-bungarotoxin (Gold and Cohen, 1981b). The bird was placed in a stereotaxic apparatus in an acoustic chamber, and electrodes for monitoring the electrocardiogram (EKG) and delivering foot-shock were attached (Cohen and Goff, 1978). The left pupil was dilated (Campbell and Smith, 1962), and a translucent contact lens of known transmission was placed over the cornea. The sutures placed during the cerebellectomy were then removed to expose the brainstem. A midline incision was made at cervical levels, and $1 \mathrm{~cm}$ of the right vagus nerve was placed on $\mathrm{Ag}-\mathrm{AgCl}$ electrodes. The nerve was bathed in a warmed suspension of petroleum jelly and mineral oil to prevent drying and cooling. Extreme care was taken with infiltration anesthesia throughout all experimental procedures to minimize discomfort. It should be noted that successful conditioning performance demands that the animal be in no distress, since discomfort is invariably reflected in high base line heart rates and a lack of conditioning.

Neuronal activity was recorded with glass micropipettes ( 8 to 12 megohms) filled with $4 \mathrm{M} \mathrm{NaCl}$. The electrode was positioned on the dorsal surface of the brainstem under visual control and was advanced by hydraulic microdrive. Vagal nerve stimulation was used to elicit field potentials in the DMN for localization of the region of cardioinhibitory neurons (Schwaber and Cohen, 1978b) and then to identify cardiac units by antidromic activation. Amplitudes of the action potentials were 100 to $500 \mu \mathrm{V}$, and noise levels were 30 to $50 \mu \mathrm{V}$.

At the end of recording, animals were sacrificed with sodium pentobarbital and perfused through an internal carotid artery with saline followed by $10 \%$ formalin. Brains were embedded in celloidon, sectioned transversely at $50 \mu \mathrm{m}$, and stained with cresylecht violet for electrode localization. In addition, the distance from the vagal stimulating elec- trodes to the brainstem was measured for computation of conduction velocity.

\section{Classes of vagal neurons and their discharge properties}

Thirty-seven of the animals received no behavioral training but were studied to assess responsiveness of vagal neurons to the light and footshock used as the conditioned and unconditioned stimuli in behavioral experiments. The responses of each unit to 10 light presentations and 10 foot-shocks were evaluated. The visual stimulus was a 50 -footlambert monocular presentation of whole field illumination with a duration of $6 \mathrm{sec}$; the mean interstimulus interval was $3.7 \mathrm{~min}$. The light source was a quartz lamp which projected through calibrated neutral density filters, an electronic shutter, and a fiberoptic bundle placed 2 to $3 \mathrm{~cm}$ from the cornea. The foot-shock consisted of $50-\mathrm{msec}$ trains of 8-msec, hiphasic, constant current pulses applied every $90 \mathrm{sec}$ to the left foot. Stimulus intensity was maintained at twice the threshold for cardiodeceleration.

For analysis, neurons were separated into three groups on the basis of axonal conduction velocities (see "Results"). To analyze visual responses, the 6-sec stimulation period was divided into a phasic segment consisting of the first 500 insec after stimulus onset and a tonic segment consisting of the remaining $5500 \mathrm{msec}$. The number of discharges in the 6 -sec base line period preceding stimulus onset and in each of the two response segments was summed over the 10 presentations, and responses were expressed as a percentage of base line activity. To analyze responses to shock, the number of discharges during the base line and 6 -sec post-shock periods was summed over the 10 presentations, and the response was expressed as a percentage of base line. Only units with maintained (base line) activity during at least $50 \%$ of the trials were used to avoid floor effects (less than $10 \%$ of the units with maintained activity). (It should be noted that the eliminated cells would not contribute to cardioacceleration, since they could show no effective decrease in discharge and no stimulus-evoked increases in discharge were ever observed in B1 units.)

Unit activity was pooled for group latency measures and for descriptive purposes. For the former, base line and response periods were divided into the smallest epochs with a mean value of $>5$ discharges/ bin in the base line period. Standardized scores were calculated for the response period relative to the base line period, and latency was defined as the time to the first bin that deviated significantly from the base line distribution.

\section{Vagal cardiac neurons}

Responses in trained animals. Fifteen of the animals received behavioral training 1 to 4 days before neurophysiological experimentation. This consisted of 100 trials of paired light and foot-shock (Cohen and Goff, 1978). Following this, $\beta$-adrenergic blockade was induced with propranolol (Cohen and Pitts, 1968) to eliminate sympathetic contributions to the $\mathrm{CK}$, and an additional 20 light-shock presentations were given to assess the vagal component of the $\mathrm{CR}$. On the following day each animal received 9 foot-shocks at current values twice threshold for tachycardia. Propranolol was then administered and the foot-shock sequence was repeated. To analyze the heart rate responses to light and shock, the R-waves of the EKG in the 6-sec period preceding the stimulus were counted (base line rate). The 6 -sec light and post-shock periods were divided into 1 -sec intervals, and the $\mathrm{R}$-waves were counted in each. When compared with base line rate, this gives the response for each interval. Unit responses were analyzed as described above.

Responses during $C R$ acquisition. These experiments involved 23 behaviorally naive birds. Animals were randomly assigned to either the associative training (conditioning) group $(n=13)$ or the nonassociative control (sensitization) group $(n=10)$. Training was initiated $10 \mathrm{~min}$ after isolation of the activity of an identified unit. For the conditioning group, whole field illumination, the conditioned stimulus (CS), was immediately followed by foot-shock, the unconditioned stimulus (US). Training consisted of 40 such light-shock pairings in a single session, the mean intertrial interval being $3.7 \mathrm{~min}$ with a range of 2.5 to 5.0 min (Cohen and Macdonald, 1971). The sensitization group also received 40 lights and 40 shocks, but these were unpaired with any two presentations (light or shock), being separated by at least $1 \mathrm{~min}$ (Cohen and Goff, 1978). The mean interval between light presentations was $3.7 \mathrm{~min}$, as in the conditioning group.

As described above, the CS was a 6-sec monocular presentation of a 50 -foot-lambert light and the US was a 500-msec foot-shock. The onset of all base line and light periods was triggered by an $\mathrm{R}$-wave of the 
FKG. The behavioral and neuronal data were analyzed as described above.

\section{Results \\ Classes of vagal neurons}

Vagal cardiac neurons in the pigeon are localized to the intermediate rostrocaudal zone of the DMN (Cohen et al., 1970; Cohen and Schnall, 1970; Schwaber and Cohen, 1978a, b). They have conduction velocities of 8.0 to $14.5 \mathrm{~m} / \mathrm{sec}$, corresponding to the B1 wave of the vagal compound action potential (Schwaber and Cohen, 1978a). Most recordings were from this zone, and no systematic effort was made to record from other areas of the DMN. While searching for cardiac neurons, antidromically activated cells were often encountered with conduction velocities outside the $B 1$ range. These non-cardiac neurons were classified by conduction velocity as $A(>15 \mathrm{~m} / \mathrm{sec})$ or B2 (2.0 to $7.5 \mathrm{~m} / \mathrm{sec}$ ) units (Schwaber and Cohen, $1978 \mathrm{~b}$ ).

A total of 5 A units, 64 B1 (cardiac) units, and 11 B2 units were studied, this distribution not being representative of the proportion of these classes in the intermediate zone due to intentional sampling bias for cardiac units. Consistent with previous anatomical (Cohen et al., 1970) and electrophysiological (Schwaber and Cohen, 1978b) findings, cardiac units were preferentially encountered in the ventral and lateral aspects of the intermediate zone. A and B2 units largely overlapped these B1 cells, although B2 cells extended slightly more caudally.

\section{Maintained activity}

Maintained activity was observed in $60 \%, 78 \%$, and $91 \%$ of the A, B1, and B2 units, respectively. Cardiac (B1) units typically discharged at $<2 \mathrm{~Hz}$ and were indistinguishable in this regard from B2 neurons. In contrast, A units discharged at approximately $5 \mathrm{~Hz}$, significantly higher than either B1 $(t(14)$ $=3.42, p<0.01)$ or $\mathrm{B} 2(t(11)=2.50, p<0.05)$ cells. Moreover, the maintained activity of $A$ units was more regular than that of $\mathrm{B} 1$ or B2 units as determined by inspection of interval histograms (Gold, 1979).

Cardiac units had no obvious respiratory rhythm, in contrast to other species (e.g., McAllen and Spyer, 1978a). The lack of a respiratory rhythm is consistent with the absence of respiratory sinus arrhythmia in the present experiments. To investigate a possible cardiac rhythm, post- $R$-wave histograms were compiled for spontaneously active neurons sampled for at least $30 \mathrm{~min}(n=38)$. Although no consistent rhythm was evident in the histograms for individual units, the pooled histogram (Fig. $1 A$ ) had a peak $31-40 \mathrm{msec}$ after the $\mathrm{R}$-wave. To evaluate the proportion of units contributing to this peak, the relative discharge rate in this time domain was calculated for each cell. As shown in Figure $1 B$, during the period of maximal activity, discharge rates were high for most units, and thus the peak appears to result from a higher probability of discharge in most cardiac units. No peaks in pooled post- $R$-wave histogram were found for A or B2 units.

\section{Responses in pretrained animals}

Visually evoked responses. Fifteen birds received behavioral training prior to recording, and all developed normal levels of conditioned cardioacceleration. Although $\beta$-adrenergic blockade at the end of training decreased the magnitude of this cardioacceleration $(F(1,28)=65.2, p<0.001)$, the persistence of a CR confirmed a vgally mediated component (Cohen and Pitts, 1968).

A pooled histogram of the responses of 13 spontaneously active cardiac units for the 6-sec control and CS periods is shown in the upper panel of Figure 2. There is clearly a decrease in discharge over the CS presentation. The lower panel of Figure 2 shows the first 1000 msec of the CS period and its preceding
A
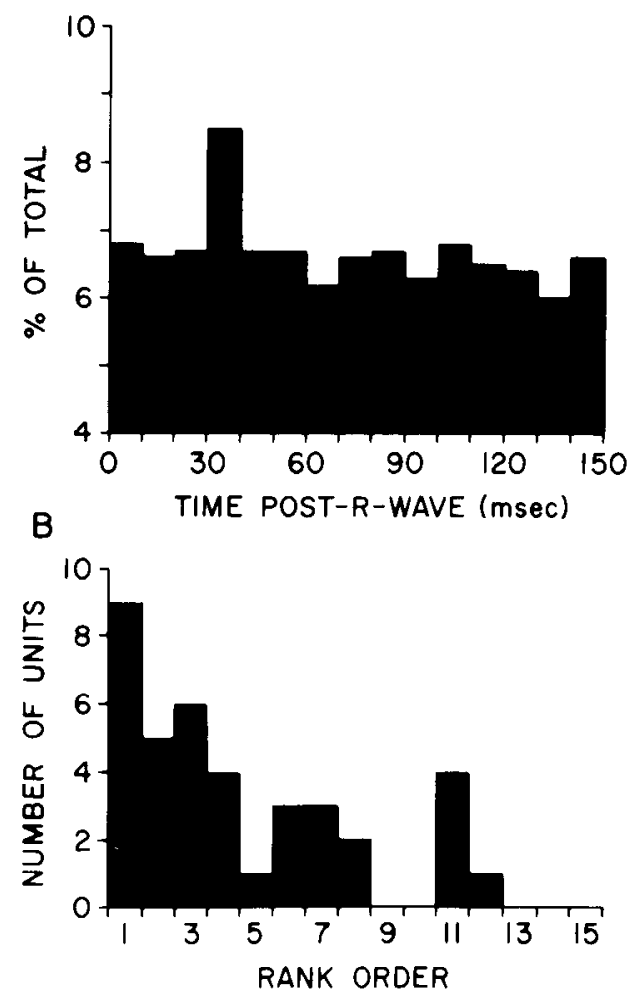

Figure 1. A, Pooled post-R-wave histogram of the activity of vagal cardiac neurons. $B$, Histogram of the rank order (relative to the other 14 bins of the post-R-wave period) of the bin 31 to $40 \mathrm{msec}$ after the $\mathrm{R}$-wave. See the text for further description.

control period. Discharge decreases from base line within 100 msec after CS onset, and this decrease is quite prominent by $200 \mathrm{msec}$. To evaluate the response latency more precisely, the pooled data were subdivided into $40-\mathrm{msec}$ epochs and activity was expressed as standardized scores relative to base line activity. Discharge first differs significantly from base line 80 to 119 msec after CS onset $(z=-2.64, p<0.005)$.

To analyze the CS-evoked responses of individual units, the CS period was divided into phasic and tonic segments (see "Materials and Methods"). Eight cells had statistically significant decreases in the phasic period and nine had them in the tonic period. For this sample, mean discharge decreased to $41 \%$ of the base line level in the phasic period $(t(10)=5.97, p<$ $0.001)$ and to $62 \%$ in the tonic period $(t(10)=4.26, p<0.001)$ (Fig. 3).

The activity of these cardiac units also varied systematically with the CR. Discharge during the tonic period was negatively correlated with heart rate (Spearman $r=-0.59, p<0.05$ ). In fact, at cardioacceleratory peaks, discharge almost totally ceased. Discharge during the phasic period also negatively correlated with the CR, but this did not reach statistical significance (Spearman $r=-0.32$, not significant). With respect to the noncardiac vagal neurons, neither A nor B2 units were responsive to the visual stimulus (Fig. 3).

Shock-evoked responses. The unconditioned response (UR) to the foot-shock is marked cardioacceleration. Although $\beta$ adrenergic blockade reduced the magnitude of this cardioacceleration $(F(1,28)=107.5, p<0.001)$, the persistence of a response after blockade indicated a vagal contribution to the UR.

The duration of the US in behavioral training was 500 msec. Since shock artifact obscured the unit records, this was reduced to $50 \mathrm{msec}$ to evaluate the effects of foot-shock on vagal discharge. The mean increase in heart rate during the 6-sec 

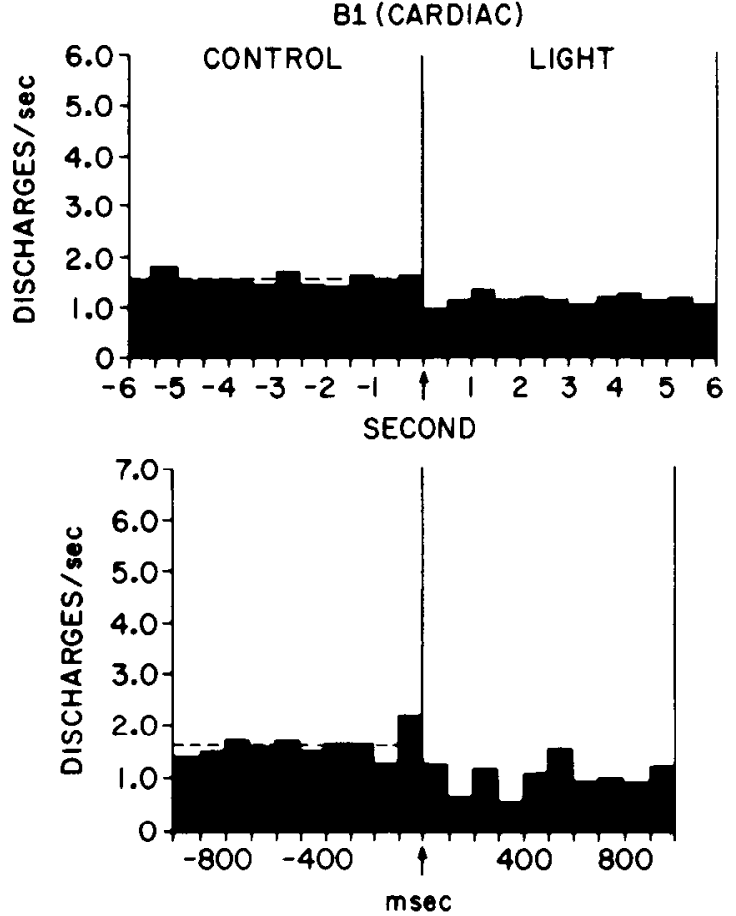

Figure 2. Peristimulus histograms of the pooled activity of B1 (cardiac) units during light presentations. The ordinate indicates the number of discharges per unit per trial per second, and the dashed lines show the mean discharge rate during the pre-light (base line) periods. The upper panel illustrates activity during the 6 -sec base line (control) and light periods in 500 -msec bins, and the lower panel illustrates activity during 1-sec periods before and after light onset in 100-msec bins. The arrows indicate light onset.

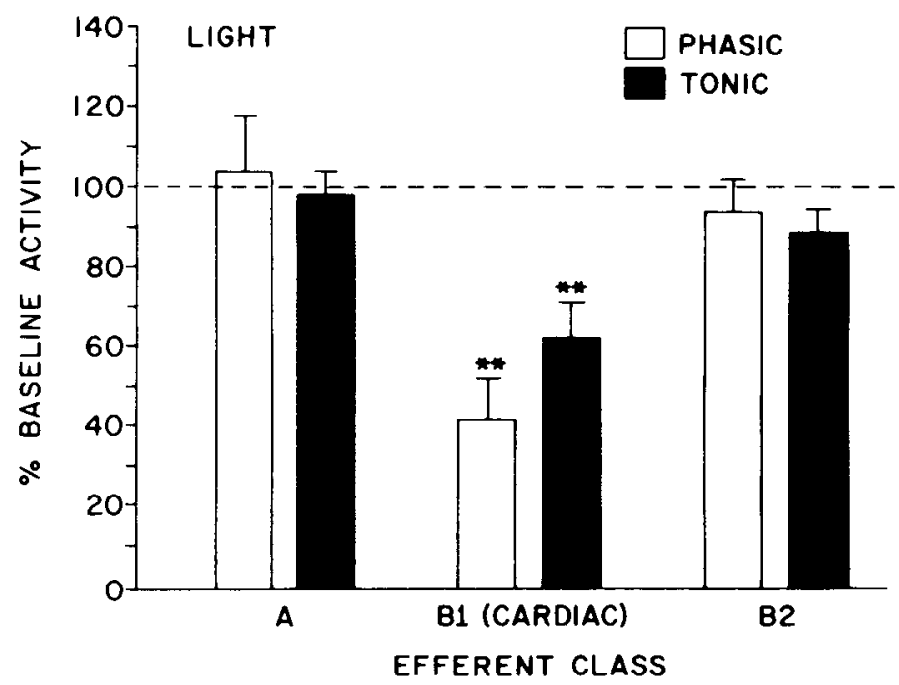

Figure 3. Discharge changes of units in the three efferent classes (A, $\mathrm{B} 1$ (cardiac), and $\mathrm{B} 2$ ) during the phasic (open bars) and tonic (solid bars) periods. The ordinate indicates the percentage of activity ( $+1 \mathrm{SE})$ in each period relative to base line rate. $* *, p<0.01$.

period following such a 50 -msec shock was 40.7 beats $/ \mathrm{min}$, with the mean heart rates of the subsamples of different unit classes not significantly differing $(F(2,26)=0.49)$.

Pooled histograms of shock-evoked responses of vagal cardiac units are shown in Figure 4. Discharge was clearly depressed throughout the 6 -sec post-shock period. Statistical analysis based upon standardized scores indicated that activity decreased significantly within $50 \mathrm{msec}$ after shock $(z=-1.75, p$ $<0.05$ ). As seen in Figure 5, discharge decreased to $70 \%$ of
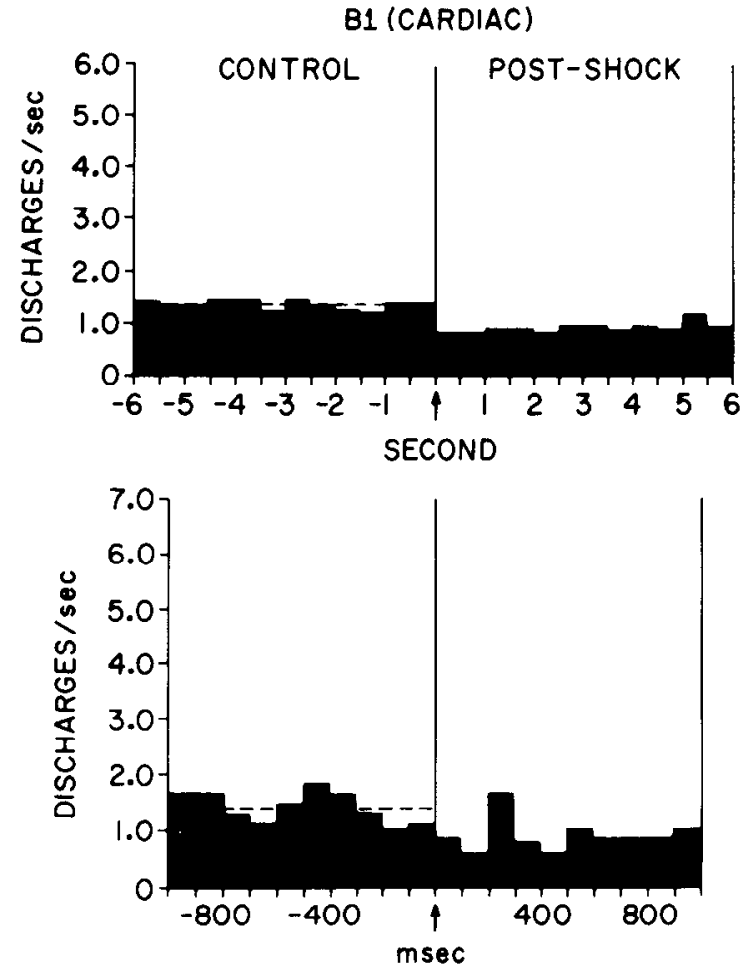

Figure 4. Peristimulus histograms of the pooled activity of B1 (cardiac) units during foot-shock presentations. The ordinate indicates the number of discharges per unit per trial per second, and the dashed lines show the mean discharge rate during the pre-shock (base line) periods. The upper panel illustrates activity during the 6 -sec base line (control) and post-shock periods in 500-msec bins, and the lower panel illustrates activity during 1 -sec periods in $100-\mathrm{msec}$ bins. The arrows indicate foot-shock.

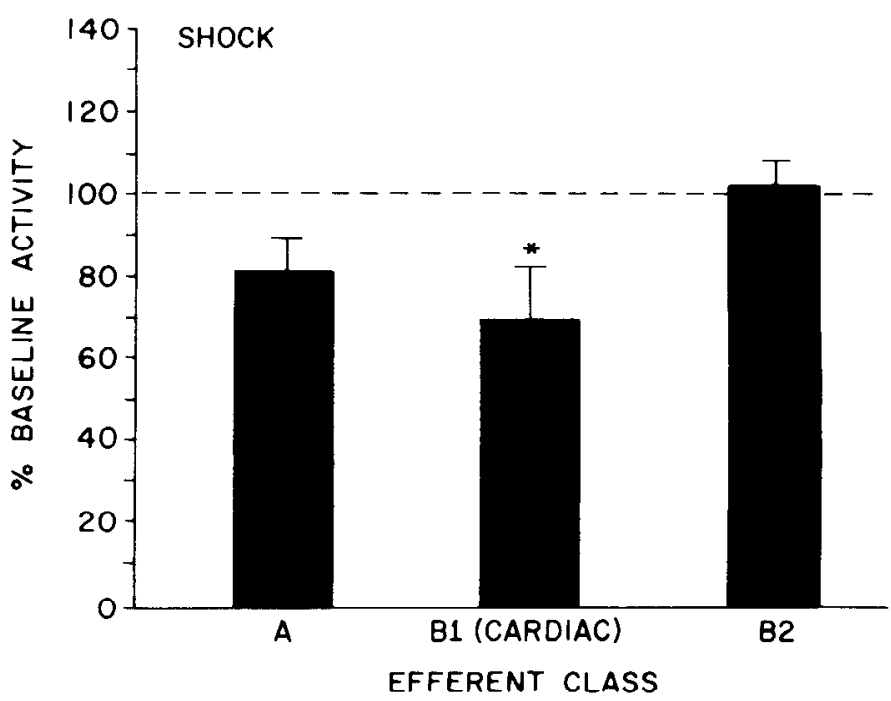

Figure 5. Discharge changes of units in the three efferent classes (A, $\mathrm{B} 1$ (cardiac), and B2) following foot-shock. The ordinate indicates the percentage of activity $( \pm 1 \mathrm{SE})$ in the post-shock relative to the base line periods. $*, p<0.05$.

base line levels $(t(7)=2.42, p<0.05)$, and this change was negatively correlated with heart rate (Spearman $r=-0.93, p$ $<0.01$ ).

With respect to noncardiac vagal neurons, the discharge of the A units appeared to decrease in response to shock (Fig. 5). However, this was not statistically significant, possibly because 
of the small sample ( $n=3$ ). The B2 units were clearly unresponsive (Fig. 5).

\section{Responses during acquisilion of the $C R$}

In 13 birds the activity of a single vagal cardiac neuron was isolated prior to training and its discharge was recorded over the acquisition of the CR. For comparison, 10 such units were studied in 10 nonassociative control birds given unpaired lights and shocks.

Heart rate. The heart rate changes for these groups are illustrated in Figure 6 . The mean heart rate response to the initial light presentation was 9.1 beats $/ \mathrm{min}$. This increased to $16.6 \mathrm{beats} / \mathrm{min}$ in the conditioning group and declined to 4.0 beats/min in the sensitization group, comparable to previous findings (Cohen and Goff, 1978; Gold and Cohen, 1981b). $\Lambda$ nalysis of variance yielded a significant groups $\times$ blocks interaction $(F(3,62)=3.94, p<0.02)$, confirming divergence of the groups and thus development of associative learning. Heart rate base lines neither differed between groups $(F(1,22)$ $=0.11$, not significant) nor changed differentially over training $(F(3,62)=0.06$, not significant $)$.

Localization and conduction velocities. The locations of the 23 cardiac neurons are shown in Figure 7 . Cells were clearly sampled throughout the intermediate rostrocaudal zone of the DMN, and there was no obvious difference in the distributions of units from the conditioning and sensitization groups. Moreover, there was no difference in the distributions of conduction velocities of the two behavioral groups $(t(21)=0.1$, not significant).

Maintained activity. Maintained (spontaneous) activity was found in $77 \%$ of the units of the conditioning group and $80 \%$ of the sensitization group. Discharge was irregular and showed a weak cardiac rhythm as described earlier. Mean discharge rates were approximately $1.5 \mathrm{~Hz}$, did not differ between the two groups $(F(1,17)=0.12$, not significant), and were not differentially affected by behavioral treatment $(F(3,47)=0.43$, not significant).

Neuronal responses. In both conditioning and sensitization groups the first light presentation preceded any foot-shocks and therefore allowed assessment of the initial response to the visual stimulus, the orienting response (Cohen and Macdonald, 1971). In both groups the discharge of the cardiac neurons decreased and heart rate increased in response to the light. Since neither the heart rate $(t(21)=0.51$, not significant) nor neuronal $(t(21)=1.58$, not significant) responses differed between the two groups, the data were pooled for further analysis of the orienting response.

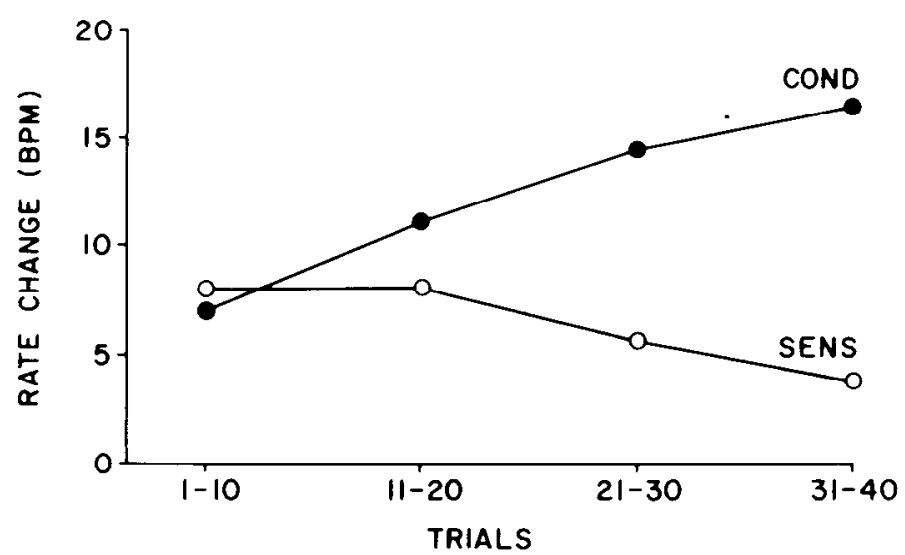

Figure 6. Mean differences between heart rate during the light and preceding base line periods for birds given conditioning (COND, $n=$ 13) or sensitization ( $S E N S, n=10$ ) paradigms. Each point represents a group mean for a block of 10 trials. $B P M$, beats per minute.
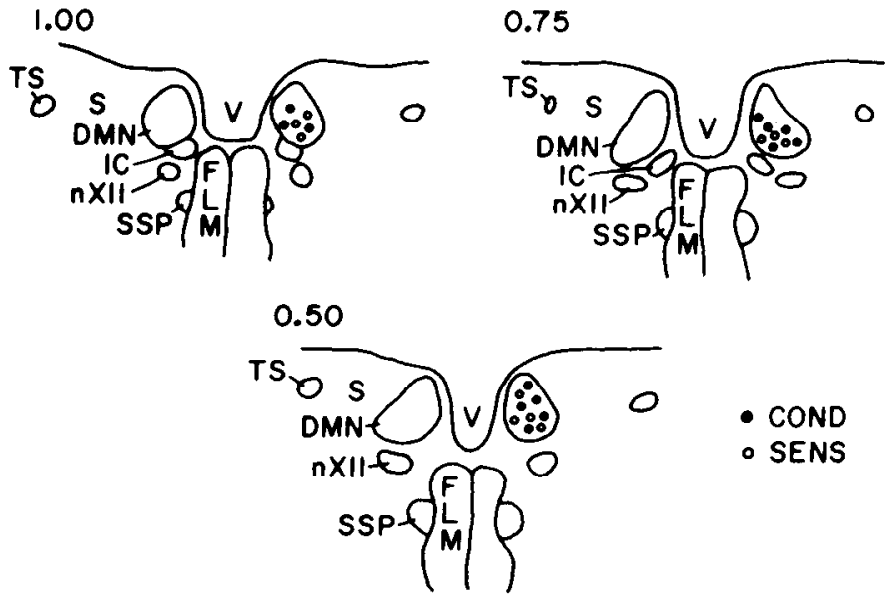

Figure 7. Schematic illustration of transverse sections of the dorsal brainstem showing the locations of recordings from vagal cardiac neurons during conditioning (COND, solid circles) or sensitization (SENS, open circles). At the upper left of each panel is the rostrocaudal level with reference to the obex $(0.00)$. DMN, dorsal motor nucleus of the vagus; $F L M$, medial longitudinal fasciculus; $I C$, nucleus intercalatus; $n X I I$, nucleus of the hypoglossal nerve; $S$, nucleus solitarius; $S S P$, nucleus supraspinalis; TS, tractus solitarius; $V$, fourth ventricle.

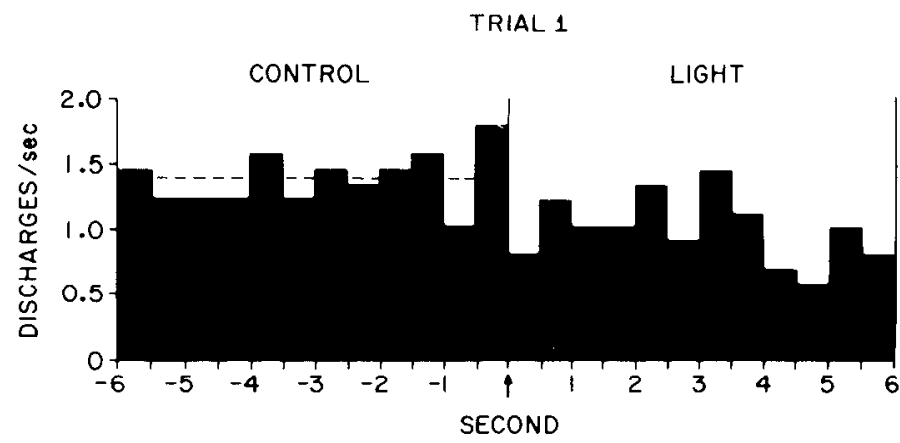

Figure 8. Peristimulus histogram of the pooled activity of vagal cardiac neurons $(n=23)$ during the initial light presentation. The ordinate indicates the number of discharges per unit per second, and the dashed line indicates the mean discharge rate during the pre-light (base line) period. The arrow indicates light onset.

A peristimulus histogram of the response of the pooled sample is shown in Figure 8. There was a significant decrease in discharge that was maintained throughout the light period $(t(22)=3.60, p<0.005)$. Accompanying this was an increase in heart rate of 9.1 beats $/ \min (t(22)=2.28, p<0.05)$.

As with heart rate responses, the light-evoked neuronal activity diverged for the two groups over training. Summary histograms for the sensitization group are shown in Figure 9. Early in training, discharge decreased as with the initial light presentation. The response then systematically attenuated, so that the neurons were effectively unresponsive by the final trial block. In contrast, units from the conditioning group showed progressive enhancement of light-evoked responses over training (Fig. 10). Thus, like the heart rate responses, neuronal activity diverges as a function of the training paradigm.

For further analysis of these data the light period was divided into phasic and tonic segments (see "Materials and Methods") and the mean discharge changes for each segment calculated (Fig. 11). As seen in Figure 10, there is little difference between the responses of the two groups early in training, but they clearly diverge over training. A significant groups effect $(F(1,17)=9.80, p<0.01)$ and groups $\times$ blocks interaction $(F(3,48)=33.0, p<0.001)$ confirmed this. Moreover, the lightevoked decrease in discharge was more prominent in the phasic 

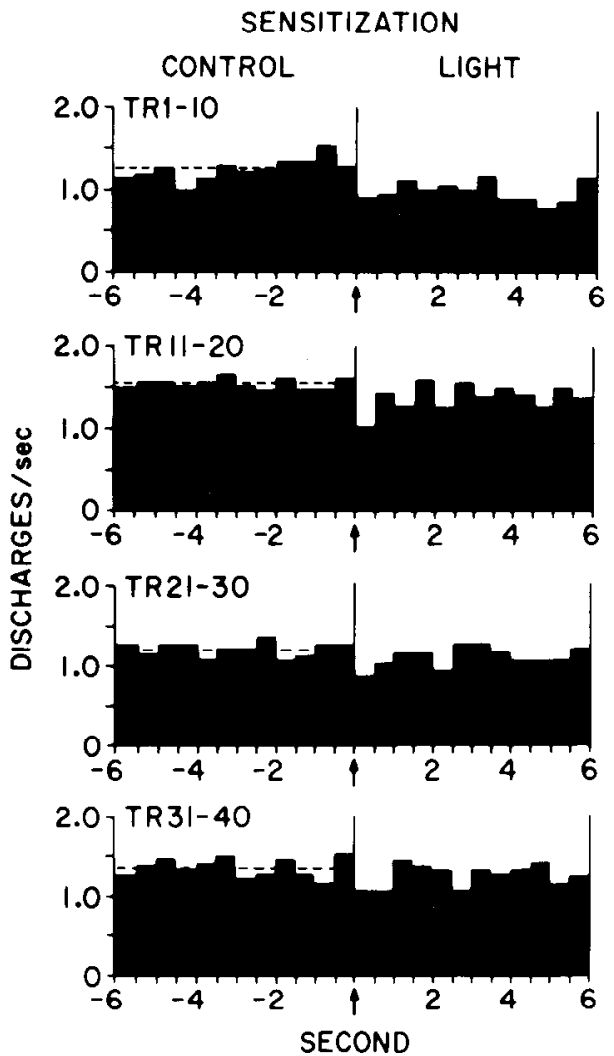

Figure 9. Peristimulus histograms of the activity of the vagal cardiac neurons $(n=10)$ during the light presentations of sensitization. Each histogram shows the pooled activity for a 10-trial block (upper left). The ordinate indicates the number of discharges per unit per trial per second, and the dashed line shows the mean discharge rate during the pre-light (base line) period. The arrows indicate light onset.

than in the tonic period $(F(1,61)=12.6, p<0.001)$. Comparison of these periods for the conditioning and sensitization groups indicated significant groups $\times$ blocks interactions for both the phasic $(F(3,47)=8.72, p<0.001)$ and tonic $(F(3,47)=12.4, p$ $<0.001)$ components, again reflecting divergence of the groups. This divergence was of sufficient magnitude that there was a significant groups effect for both phasic $(F(1,17)=5.69, p<$ $0.05)$ and tonic $(F(1,17)=10.3, p<0.01)$ response periods. A comparison of Figures 6 and 11 indicates the close parallel between these changes in the discharge of vagal cardiac neurons and the development of the heart rate CR.

The significant group difference in the phasic period establishes that short-latency responses are affected by training. To estimate more precisely the latency of this differential change, the histograms for 10-trial blocks were subdivided into 40 -msec epochs, and standardized scores relative to base lines were calculated for the first 200 msec. The standardized scores for the first two and the last two training blocks were then averaged (Fig. 12). Early in training (Fig. 12A), when there are no group differences in behavioral or neuronal responses to the light, the decrease in discharge first reaches significance 120 to $159 \mathrm{msec}$ after light onset in both conditioning $(z=-1.82, p<0.05)$ and sensitization $(z=-1.57, p<0.05)$ groups. However, later in training when the groups differ with respect to behavioral and neuronal responses, the latency of the decreased discharge in the conditioning group now reaches significance by 80 to 119 msec $(z=-1.40, p<0.05)$, whereas in the sensitization group the latency has increased to 160 to $199 \mathrm{msec}(z=-1.40, p<$ 0.05 ).

Since the CS-evoked responses of vagal cardiac neurons were studied after conditioning in one group of birds and during CR
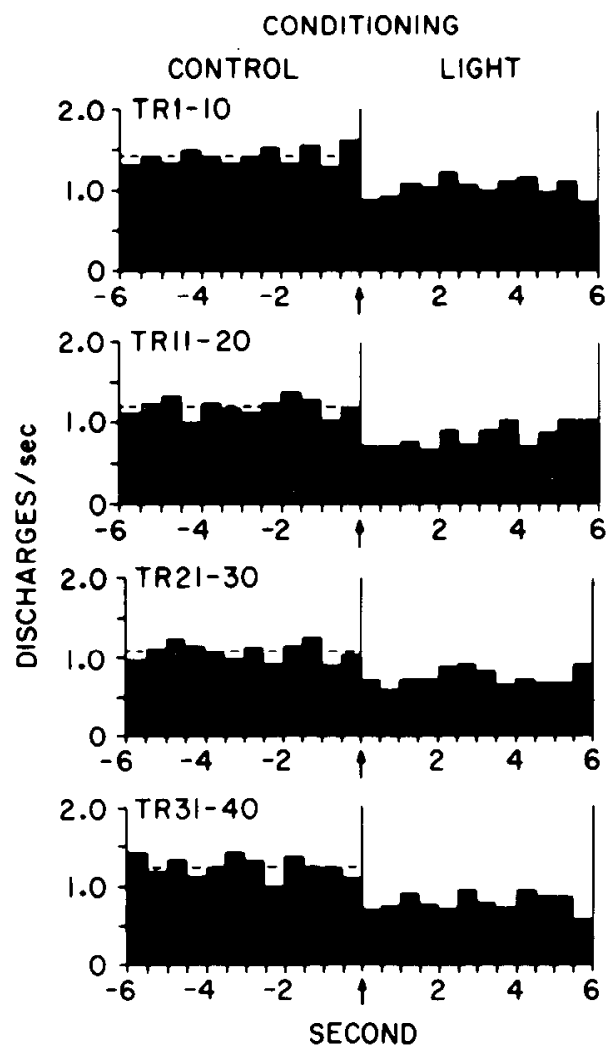

Figure 10. Peristimulus histograms of the activity of the vagal cardiac neurons $(n=13)$ during the light (CS) presentations of conditioning. See the legend to Figure 9 for further details.
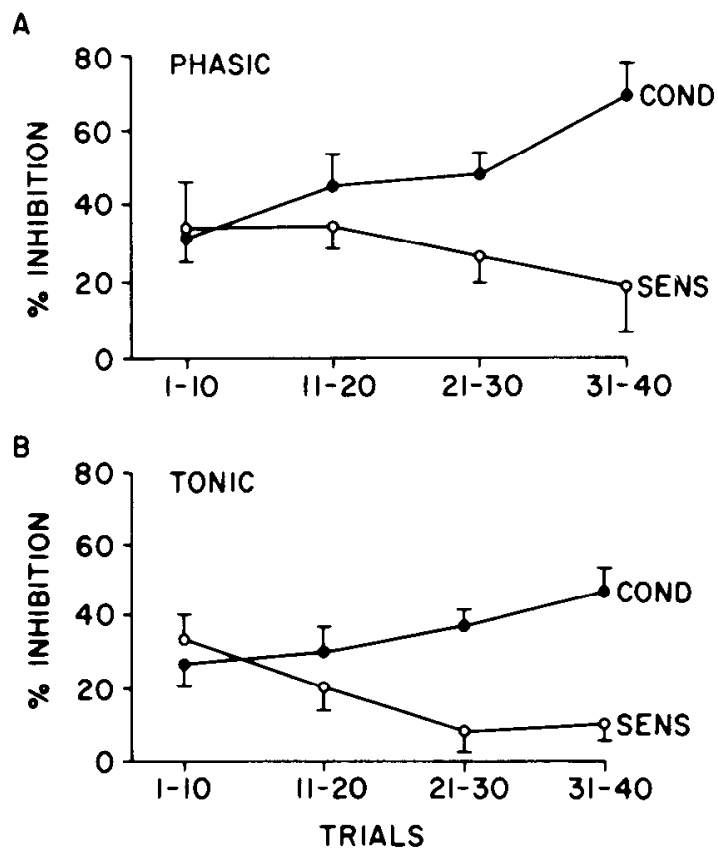

Figure 11. Mean discharge changes of vagal cardiac neurons during conditioning (COND, $n=10$ ) or sensitization (SENS, $n=8$ ). The ordinate indicates the percentage of decrease in discharge relative to the base line. Each point represents a group mean for a block of 10 trials; the error bars represent $1 \mathrm{SE}$. $A$, Phasic response period; $B$, tonic response period. 
A

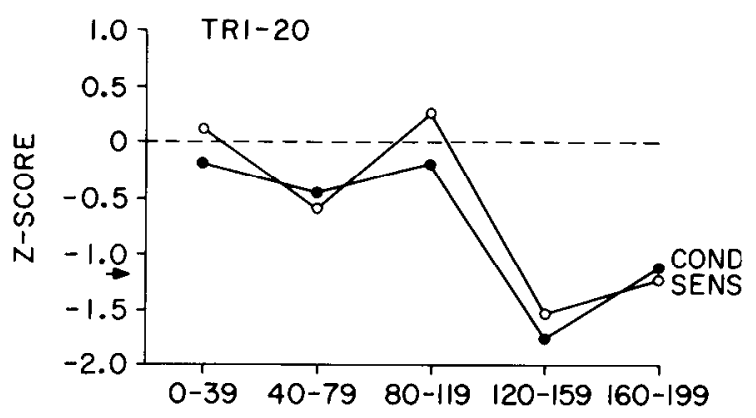

B

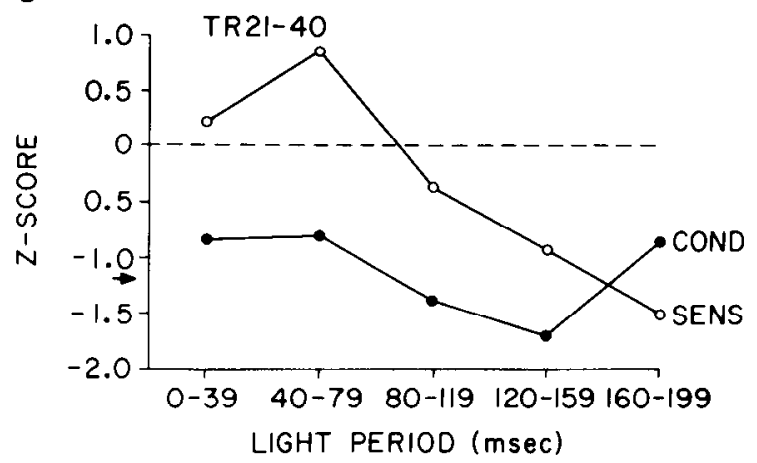

Figure 12. Change in the response latency of vagal cardiac neurons during conditioning (COND) or sensitization (SENS). Each point represents a mean for two 10-trial blocks. The ordinate indicates the standardized score for each 40 -msec bin relative to the base line distribution, and the arrow indicates the standardized score $(z=-1.17)$ below which values differ significantly from the base line at the 0.05 level. $A$, 'I'rials ('TR) 1 to $20 ; B$, trials 21 to 40 .

acquisition in another, responses at asymptotic performance could be compared for the two groups. Such a comparison demonstrated that both the phasic and tonic responses of the two neuronal populations were equivalent after training. Also, the response latencies of the two populations did not differ. Given this, the data from the pretrained birds were pooled with those from the final acquisition block of the longitudinal sample to gain further resolution in estimating the latency of the CSevoked response (Fig. 13). For this pooled sample, the CSevoked change in activity first reached significance 80 to 99 msec after stimulus onset $(z=-1.76, p<0.05)$.

\section{Discussion}

\section{Classes of vagal motoneurons}

Three classes of neurons in the intermediate zone of the DMN were distinguished on the basis of axonal conduction velocities. These corresponded to waves previously described for the compound vagal action potential (Schwaber and Cohen, 1978 a) and the antidromically evoked field potential in the DMN (Schwaber and Cohen, 1978b). Whereas the B1 units are known to be cardiac neurons (Schwaber and Cohen, 1978b), the peripheral targets of the $A$ and $B 2$ units have not been determined. However, recent anatomical data suggest they would innervate the secretory stomach (proventriculus) or caudal abdominal organs (Katz, 1981).

The B1 and B2 cells could not be distinguished on the basis of maintained activity, but both classes were discriminable from the A neurons which discharged at higher, more regular rates. Differences in the maintained activity of different classes of vagal neurons are well established. For example, Jewett (1964) distinguished seven classes in cervical vagal recordings, and different discharge patterns have been reported for the vagal innervations of the digestive tract (Iggo and Leek, 1976; Davison and Grundy, 1978), respiratory tract (Eyzaguirre and Taylor, 1963; Widdicombe, 1966), and thoracic viscera (McAllen and Spyer, 1978a).

Responses to exteroceptive stimulation also discriminated among the three classes of vagal neurons. Visual stimulation markedly decreased the activity of the cardiac units, while not affecting the $A$ and B2 units. This suggests differential inputs to the various classes of vagal neurons. Since visual stimulation also evokes cardioacceleration, the decreased activity of the vagal cardiac neurons confirms that release of vagal inhibition of the heart contributes to this tachycardia.

Foot-shock also evoked differential responses, decreasing the discharge of the B1 units but not affecting the B2 cells. The activity of the $A$ units also decreased, but not significantly so. Again, one would anticipate decreased activity of the cardiac neurons, since foot-shock evokes cardioacceleration. Similar results have been reported in studies that have assessed vagal responses to somatosensory stimuli. In cats, noxious cutaneous stimulation inhibits vagal cardiac discharge (Kaufman et al., 1977), and electrical stimulation of somatic nerves in dogs decreases the activity of vagal cardiac units (Iriuchijima and Kumada, 1963).

Finally, the B1 cells had a weak cardiac rhythm which was not evident in A and B2 units; none of the classes showed a respiratory rhythm. In contrast, in the chloralose-anesthetized cat, few vagal cardiac neurons have maintained activity, but many of those that are active have marked cardiac and respiratory rhythms (Kunze, 1972; McAllen and Spyer, 1976, 1978a, b; Ciriello and Calaresu, 1980). However, in this preparation there is little vagal tone and marked respiratory sinus arrhythmia, whereas in our preparation vagal tone is high and no respiratory sinus arrhythmia is evident. It is thus possible that chloralose accounts for the marked cardiac and respiratory rhythms reported for cats. Furthermore, it has been shown that chloralose sensitizes the baroreceptor reflex (Cox and Bagshaw,

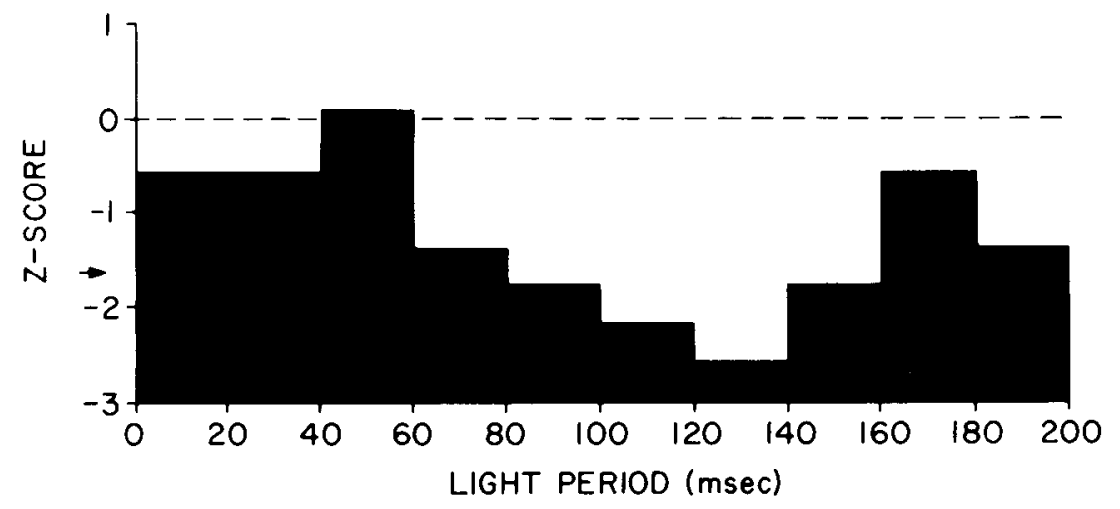

Figure 13. Post-stimulus histogram showing the response latency to the CS of vagal cardiac neurons ( $n=$ 30) during asymptotic performance. Data were pooled from conditioning animals in the last trial block of training (trials 31 to 40 ) and from pretrained animals. The ordinate indicates the standardized score for each 20 -msec bin relative to the base line distribution, and the arrow indicates the standardized score $(z=-1.65)$ below which values differ significantly from the base line at the 0.05 level. 
1979). This may enhance the vagal cardiac rhythm which is influenced by baroreceptor input (McAllen and Spyer, 1978b; Kunze, 1980). In support of this hypothesis, vagal cardiac activity in decerebrate cats infrequently shows a cardiac rhythm and is uncorrelated with respiration (Schwartz et al., 1972). Moreover, chloralose-anesthetized dogs have tonically active vagal cardiac neurons which show both cardiac and respiratory rhythms (Iriuchijima and Kumada, 1964; Kollai and Koizumi, 1981).

In summary, the discharge properties of B1 neurons are consistent with their presumed cardioinhibitory function. Most are tonically active, and this would account for the high resting vagal tone in the pigeon (Cohen and Pitts, 1968). Moreover, changes in maintained activity are generally associated with reciprocal changes in heart rate. During stimulus-evoked cardioacceleration or spontaneous tachycardic episodes, the vagal cardiac neurons show a decrease in activity. On the other hand, stimuli that do not have a chronotropic effect in this preparation, such as the activation of pulmonary receptors during inspiration, do not affect the activity of the vagal cardiac neurons.

\section{Activity of vagal cardiac neurons associated with conditioned heart rate change}

The primary objective of this study was to characterize the discharge of vagal cardiac neurons during classically conditioned change in heart rate. The behavioral data clearly indicated that the conditioning paradigm established associative learning, consistent with previous reports (Cohen and Goff, 1978; Gold and Cohen, 1981b). With regard to neuronal activity, vagal cardiac discharge systematically changed over training, closely paralleling the change in heart rate. More specifically, the initial CS-evoked decrease in vagal activity became increasingly pronounced over conditioning, whereas it attenualed over unpaired light and shock presentations. These findings confirm the previous suggestion based on behavioral data (Cohen and Pitts, 1968) that the vagal cardiac innervation contributes directly to the cardioacceleratory CR. Since the sympathetic outflow increases in response to the CS (Cabot, 1976; Cohen, 1982), it is clear that the sympathetic and parasympathetic innervations act synergistically to produce the $\mathrm{CR}$, analogous to their behavior in response to many chronotropic stimulus situations (Schwartz et al., 1972; Kollai and Koizumi, 1981).

The finding that the vagal cardiac neurons respond to the visual stimulus prior to training indicates a pre-existing, CSresponsive pathway from the eye to the heart. It is noteworthy that the excitability of the motoneurons involved in the conditioned nictitating membrane response of the rabbit is also affected by the CS prior to training (Young et al., 1976; Berthier and Moore, 1983). Indeed, it is possible that in vertebrate systems initial CS responsiveness of the pathways mediating conditioning (including the motoneurons) may be a frequent, if not general, finding. Such responsiveness of the vagal cardiac neurons suggests that the parasympathetic cardiac innervation contributes to the cardioacceleratory orienting response of the pigeon (Cohen and Macdonald, 1971). With unpaired lights and shocks the orienting response habituates, and this is reflected in the attenuation of the light-evoked response of vagal cardiac neurons. In contrast, associative training enhances the neuronal response, suggesting that the training-induced modification involves modulation at one or more sites along an existing pathway.

Analogous to the CS-evoked response of the cardiac sympathetics (Cabot, 1976; Cohen, 1982), the vagal response consists of phasic and tonic components. The phasic decrease in discharge has a latency of approximately $120 \mathrm{msec}$ which shortens to approximately $80 \mathrm{msec}$ as conditioning progresses. Thus, the vagal innervation mediates the shortest latency component of the CR, as had been concluded earlier from behavioral findings (Cohen and Pitts, 1968). At asymptotic response levels the phasic component is maximal at 120 to $140 \mathrm{msec}$, at which point it begins returning toward the base line. However, discharge remains below base line levels throughout the remainder of the CS period; this is designated the tonic component of the vagal response and is consistent with the long-duration heart rate change in animals with $\beta$-blockade. The phasic response is greater than the tonic at all phases of training, although both augment systematically over acquisition of the CR. In the nonassociative condition, both the phasic and tonic components attenuate systematically, the tonic component more prominently.

The latency of the phasic component is sufficiently short that it occurs before any cardiovascular response. Therefore, feedback from the periphery cannot contribute to this component of the response. The temporal properties of the tonic component do not exclude this possibility. Since peripheral feedback during cardioacceleration would be expected to excite vagal cardiac neurons, it might reduce the magnitude of the tonic component. On the other hand, rate-limiting reflexes could well be uncoupled by the CS-evoked input, as has been demonstrated for some cardiovascular responses (Humphreys et al., 1971).

In summary, the present findings demonstrate a number of important points with respect to the organization of the neuronal circuitry in this conditioning model. (a) A vagal contribution to the CR is confirmed, and this acts synergistically with the sympathetic contribution. (b) Vagal cardiac neurons are initially responsive to the CS, and the effect of associative training is to enhance this response. (c) Training-related changes in the discharge of vagal cardiac neurons closely parallel the behavioral response. (d) The response of vagal cardiac neurons has phasic and tonic components, the phasic component being most prominent and showing greater change with training. (e) The latency of the vagal response is surprisingly short, suggesting that the system may be more amenable to analysis than might have been anticipated.

\section{References}

Alkon, D. L. (1982) A biophysical basis for molluscan associative learning. In Conditioning, C. D. Woody, ed., pp. 147-170, Plenum Press, New York.

Berthier, N. E., and J. W. Moore (1983) The nictitating membrane response: An electrophysiological study of the abducens nerve and nucleus and the accessory abducens nucleus in rabbit. Brain Res. 258: 201-210.

Cabot, J. B. (1976) Anatomical and electrophysiological analysis of the sympathomotor pathway mediating heart rate conditioning in the pigeon. Ph.D. thesis, University of Virginia, Charlottesville.

Campbell, H. S., and J. L. Smith (1962) The pharmacology of the pigeon pupil. Arch. Opthalmol. 67: 141-144.

Ciriello, J., and F. R. Calaresu (1980) Distribution of vagal cardioinhibitory neurons in the medulla of the cat. Am. J. Physiol. 238: R57R64.

Cohen, D. H. (1974a) The neural pathways and informational flow mediating a conditioned autonomic response. In Limbic and Autonomic Nervous Systems Research, L. V. DiCara, ed., pp. 223-275, Plenum Press, New York.

Cohen, D. H. (1974b) Analysis of the final common path for heart rate conditioning. In Cardionascular Psychophysinlogy, P. A. Obrist, A. H. Black, J. Brener, and L. V. DiCara, eds., pp. 117-135, Aldine Publishing Co., Chicago.

Cohen, D. H. (1980) The functional neuroanatomy of a conditioned response. In Neural Mechanisms of Goal-Directed Behavior and Learning, R. F. Thompson, L. H. Hicks, and V. B. Shvyrkov, eds., pp. 283-302, Academic Press, Inc., New York.

Cohen, D. H. (1982) Central processing time for a conditioned response in a vertebrate model system. In Conditioning, C. D. Woody, ed., pp. 517-534, Plenum Press, New York.

Cohen, D. H. (1984) Identification of vertebrate neurons modified 
during learning: Analysis of sensory pathways. In Primary Neural Substrates of Learning and Behavioral Change, D. L. Alkon and J. Farley, eds., pp. 129-154, Cambridge University Press, Cambridge, England.

Cohen, D. H., and D. M Goff (1978) Conditioned heart rate change in the pigeon: Analysis and prediction of acquisition patterns. Physiol. Psychol. 6: 127-141.

Cohen, D. H., and R. L. Macdonald (1971) Some variables affecting orienting and conditioned heart-rate responses in the pigeon. $\mathrm{J}$. Comp. Physiol. Psychol. 74: 123-133.

Cohen, D. H., and L. H. Pitts (1968) Vagal and sympathetic components of conditioned cardioacceleration in the pigeon. Brain Res. 9: $15-31$.

Cohen, D. H., and A. M. Schnall (1970) Medullary cells of origin of vagal cardioinhibitory fibers in the pigeon. II. Flectrical stimulation of the dorsal motor nucleus. J. Comp. Neurol. 140: 321-342.

Cohen, D. H., A. M. Schnall, R. L. Macdonald, and L. H. Pitts (1970) Medullary cells of origin of vagal cardioinhibitory fibers in the pigeon. I. Anatomical studies of peripheral vagus nerve and the dorsal motor nucleus. J. Comp. Neurol. 140: 299-320.

Cox, R. H., and R. J. Bagshaw (1979) Effects of anesthesia on the baroreflex control of heart rate in chronically instrumented dogs. Fed. Proc. 38: 1320 .

Davison, J. S., and D. Grundy (1978) Modulation of single vagal efferent fibre discharge by gastrointestinal afferents in the rat. $\mathrm{J}$. Physiol. (Lond.) 284: 69-82.

Eyzaguirre, C., and J. R. Taylor (1963) Respiratory discharge of some vagal motoneurons. J. Neurophysiol. 26: 61-78.

Fold, M R. (1979) Analysis of the vagal involvement in conditioned heart rate change in the pigeon, Columba livia. Ph.D. thesis, University of Virginia, Charlottesville.

Gold, M. R., and D. H. Cohen (1981a) Modification of the discharge of vagal cardiac neurons during conditioned heart rate change. Science 214: $345-347$.

Gold, M. R., and D. H. Cohen (1981b) Ileart rate conditioning in the pigeon immobilized with $\alpha$-bungarotoxin. Brain Res. 216: 163-172.

Humphreys, P. W., N. Joels, and R. M. McAllen (1971) Modification of the reflex response to stimulation of carotid sinus baroreceptors during and following stimulation of the hypothalamic defense areas in the cat. J. Physiol. (Lond.) 216: 461-482.

Iggo, A., and B. F. Leek (1967) An electrophysiological study of single vagal efferent units associated with gastric movements in sheep. $J$. Physiol. (Lond.) 191: 177-204.

Iriuchijima, J., and M. Kumada (1963) Efferent cardiac vagal discharge of the dog in response to electrical stimulation of sensory nerves. Jpn. J. Physiol. 13: 599-605.

Iriuchijima, J., and M. Kumada (1964) Activity of single vagal fibers efferent to the heart. Jpn. J. Physiol. 14: 479-487.

Jewett, D. L. (1964) Activity of single efferent fibres in the cervical vagus nerve of the dog, with special reference to possible cardioinhibitory fibres. J. Physiol. (Lond.) 175: 321-357.

Kandel, E. R., and J. H. Schwartz (1982) Molecular biology of learning: Modulation of transmitter release. Science 218: 433-443.
Katz, D. M. (1981) Anatomical organization of the dorsal motor nucleus of the vagus nerve and nuclei of the tractus solitarius in the pigeon, Columba livia. Cytoarchitecture and peripheral visceral connections. Ph.D. thesis, State University of New York at Stony Brook, Stony Brook.

Kaufman, A., A. Sato, Y. Sato, and H. Sugimoto (1977) Reflex changes in heart rate after mechanical and thermal stimulation of the skin at various segmental levels in cats. Neuroscience 2: 103-109.

Kollai, M., and K. Koizumi (1981) Cardiovascular reflexes and interrelationships between sympathetic and parasympathetic activity. $J$. Auton. Nerv. Syst. 4: 135-148.

Kunze, D. L. (1972) Reflex discharge patterns of cardiac vagal efferent fibres. J. Physiol. (Lond.) 222: 1-15.

Kunze, D. L. (1980) Regulation of activity of cardiac vagal motoneurons. Fed. Proc. 39; 2513-2518.

McAllen, R. M., and K. M. Spyer (1976) The location of cardiac vagal preganglionic motoneurones in the medulla of the cat. J. Physiol. (Lond.) 258: 187-204.

McAllen, R. M., and K. M. Spyer (1978a) Two types of vagal preganglionic motoneurones projecting to the heart and lungs. J. Physiol. (Lond.) 282: 353-364.

McAllen, R. M., and K. M. Spyer (1978b) The baroreceptor input to cardiac vagal motoneurones. J. Physiol. (Lond.) 282: 365-374.

Schwaber, J. S., and D. H. Cohen (1978a) Electrophysiological and electron microscopic analysis of the vagus nerve of the pigeon, with particular reference to the cardiac innervation. Brain Res. 147: 6578.

Schwaber, J. S., and D. H. Cohen (1978b) Field potential and single unit analysis of the avian dorsal motor nucleus of the vagus and criteria for identifying vagal cardiac cells of origin. Brain Res. 147: 79-90.

Schwartz, P. J., M. Pagani, F. Iombardi, G. Recordati, A. Malliani, and A. M. Brown (1972) Reflex changes in cardiac vagal efferent nervous activity elicited by stimulation of afferent fibres in the cardiac sympathetic nerves. Brain Res. 42: 482-485.

Thompson, R. F., T. W. Berger, S. D. Berry, G. A. Clark, R. N. Ketter, D. G. Lavond, M. D. Mauk, D. A. McCormick, P. R. Solomon, and D. J. Weisz (1982) Neuronal substrates of learning and memory: Hippocampus and other structures. In Conditioning, C. D. Woody, ed., pp. 115-129, Plenum Press, New York.

Tsukahara, N. (1982) Classical conditioning mediated by the red nucleus in the cat. In Conditioning, C. D. Woody, ed., pp. 223-231, Plenum Press, New York.

Warner, H. R., and A. Cox (1962) A mathematical model of heart rate control by sympathetic and vagus efferent information. J. Appl. Physiol. 17: 349-355.

Widdicombe, J. G. (1966) Action potentials in parasympathetic and sympathetic fibres to the trachea and lungs of dogs and cats. J. Physiol. (Lond.) 186: 56-88.

Young, R. A., C. F. Cegavske, and R. F. Thompson (1976) Toneinduced changes in excitability of abducens motoneurons and of the reflex path of nictitating membrane responses in rabbit (Oryctolagus cuniculus). J. Comp. Physiol. Psychol. 90: 424-434. 